\title{
Aerodynamic drag of modern soccer balls
}

\author{
Takeshi Asai ${ }^{1 *}$ and Kazuya Seo ${ }^{2}$
}

\begin{abstract}
Soccer balls such as the Adidas Roteiro that have been used in soccer tournaments thus far had 32 pentagonal and hexagonal panels. Recently, the Adidas Teamgeist II and Adidas Jabulani, respectively having 14 and 8 panels, have been used at tournaments; the aerodynamic characteristics of these balls have not yet been verified. Now, the Adidas Tango 12, having 32 panels, has been developed for use at tournaments; therefore, it is necessary to understand its aerodynamic characteristics. Through a wind tunnel test and ball trajectory simulations, this study shows that the aerodynamic resistance of the new 32-panel soccer ball is larger in the high-speed region and lower in the middle-speed region than that of the previous 14- and 8-panel balls. The critical Reynolds number of the Roteiro, Teamgeist II, Jabulani, and Tango 12 was $\sim 2.2 \times 10^{5}$ (drag coefficient, $C_{d} \approx 0.12$ ), $\sim 2.8 \times 10^{5}$ $\left(C_{d} \approx 0.13\right), \sim 3.3 \times 10^{5}\left(C_{d} \approx 0.13\right)$, and $\sim 2.4 \times 10^{5}\left(C_{d} \approx 0.15\right)$, respectively. The flight trajectory simulation suggested that the Tango 12, one of the newest soccer balls, has less air resistance in the medium-speed region than the Jabulani and can thus easily acquire large initial velocity in this region. It is considered that the critical Reynolds number of a soccer ball, as considered within the scope of this experiment, depends on the extended total distance of the panel bonds rather than the small designs on the panel surfaces.
\end{abstract}

Keywords: Aerodynamic, Drag, Ball, Soccer, Critical reynolds number, Fright trajectory, Sport

\section{Introduction}

Following Thompson's (1910) pioneering study of golf balls, studies of the aerodynamic characteristics of golf balls have focused on their drag coefficient $\left(C_{d}\right)$ values and their dimpled shapes (Bearman and Harvey 1976; Davies 1949; Smits and Ogg 2004). Similarly, other studies have investigated the flight of cricket balls (Mehta et al. 1983), baseballs (Watts and Sawyer 1975; Watts and Ferrer 1987; LeRoy et al. 2001; Nathan et al. 2008), tennis balls (Štěpánek 1988; Zayas 1986), and volleyballs (Wei et al. 1988); many of these have been reviewed by Mehta (1985). Previous studies of the aerodynamic characteristics of soccer balls have focused on traditional 32-panel balls such as the Adidas Roteiro, where each panel is a pentagon or a hexagon (Bray and Kerwin 2003; Asai et al. 2007; Goff and Carré 2009). In recent times, though, balls featuring a reduced number of panels have been used at major tournaments. For example, the Adidas Teamgeist II, the official match ball of the 2008 Beijing Olympic Games, has 14 panels, and the Adidas Jabulani, the official match ball of

\footnotetext{
* Correspondence: asai@taiiku.tsukuba.ac.jp

${ }^{1}$ Institute of Health and Sport Sciences, B207, University of Tsukuba, Tsukuba 305-8574, Japan

Full list of author information is available at the end of the article
}

the South Africa 2010 FIFA World Cup, has only 8 panels. Despite this trend toward fewer panels (Asai et al. 2007), few studies (Asai and Kamemoto 2011) have focused on the aerodynamic characteristics of these balls. Having said that, the Adidas Tango 12, the new soccer ball used at the UEFA Euro 2012 and the 2012 London Olympics, has 32 panels having a new shape and a curved design. Therefore, it is necessary to understand the aerodynamic characteristics of this new ball.

In this study, we conducted a steady-state analysis of the newest soccer ball-the Adidas Tango 12 (32 panels)—and conventional soccer balls-the Adidas Roteiro (32 panels), Adidas Teamgeist II (14 panels), and Adidas Jabulani (8 panels)-through a wind tunnel experiment, and we clarified the drag coefficient and critical Reynolds number. A simple 2D flight trajectory simulation was conducted based on the drag coefficient, and the effects of the drag characteristics on the flight distance and flight trajectory were examined. The relationship between the critical Reynolds number and the extended total distances of the panel bonds of the soccer balls was examined, and the two were shown to have a high degree of correlation. 


\section{Methods}

Wind tunnel test

We measured the aerodynamic forces acting on different types of balls in a low-speed wind tunnel having a $0.7 \mathrm{~m} \times$ $0.7 \mathrm{~m}$ rectangular cross section (turbulence level: $\leq 1 \%$ ). Four full-sized official FIFA soccer balls were tested: the conventional balls-the Adidas Roteiro (smooth surface with 32 pentagonal and hexagonal panels, used at UEFA Euro 2004), the Adidas Teamgeist II (small protuberances with 14 panels, used at the 2008 Beijing Olympic Games), and the Adidas Jabulani (small ridges or protrusions with 8 panels, used at the South Africa 2010 FIFA World Cup)and the newly designed ball-the Adidas Tango 12 (small grip texture with 32 panels, used at UEFA Euro 2012 and the 2012 London Olympic Games) (Figure 1).

Each soccer ball was attached to a stainless steel rod (Figure 2). In the wind tunnel experiment, the position of the support rod relative to the bluff body is important; therefore, we had to select an appropriate support method. In the experiment, we provided support from the rear (Achenbach, 1972), which we considered to have a comparatively smaller effect on peeling off of the boundary layer at the ball's surface. We also measured the aerodynamic forces acting on the ball's support without the ball. (The ball's support does not make contact with the dummy ball.) This value was subtracted from the aerodynamic forces acting on the ball with the support. Data were acquired over a period of $8.192 \mathrm{~s}$ using a threecomponent strut-type balance (LMC-3531-50NS; Nissho Electric Works Co., Ltd.), and they were recorded on a personal computer using an A/D converter board (sampling rate: $1000 /$ s). Each ball was set to be geometrically

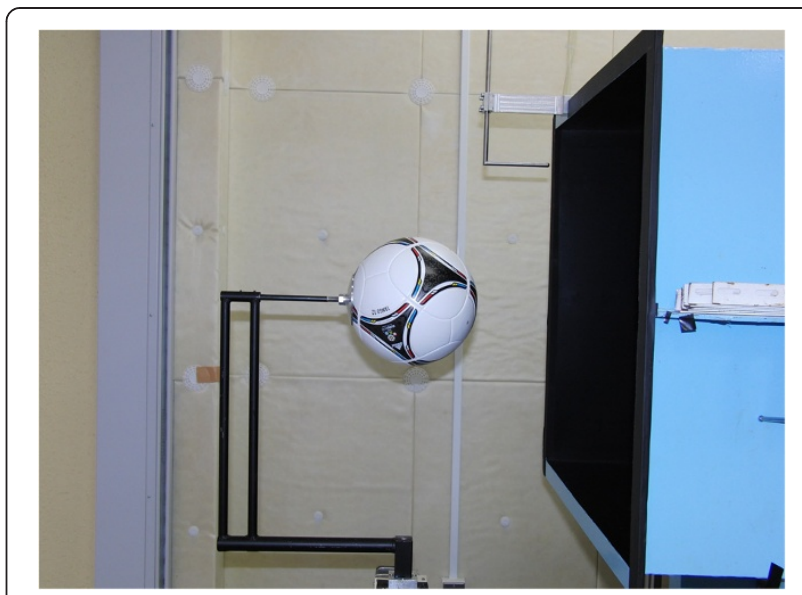

Figure 2 Experimental setup with wind tunnel.

symmetrical; therefore, the ball panels were asymmetrical in the vertical direction.

The aerodynamic forces were measured at wind speeds, $U$, of 7-30 $\mathrm{m} / \mathrm{s}$. The force acting in the direction opposite to that of the wind $(\operatorname{drag} D)$ was calculated from the experimental data collected under different conditions. The measured aerodynamic forces were then used to calculate $C_{d}$ using the following equation:

$$
C_{d}=\frac{D}{\frac{1}{2} \rho U^{2} A}
$$

Here, $\rho$ is the density of air $\left(1.2 \mathrm{~kg} / \mathrm{m}^{3}\right) ; U$, the flow velocity $(\mathrm{m} / \mathrm{s})$; and $A$, the projected area $\left(\mathrm{m}^{2}\right)$ of the soccer ball.
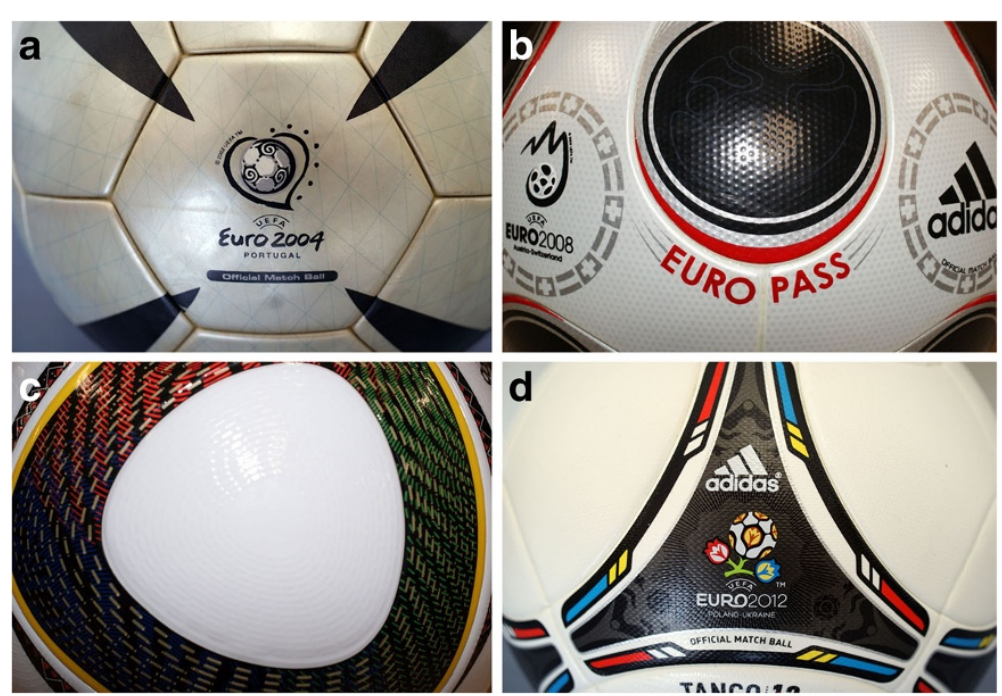

Figure 1 Photographs of soccer balls. (a) Adidas Roteiro: smooth surface with 32 pentagonal and hexagonal panels. (b) Adidas Teamgeist II: small protuberances with 14 panels. (c) Adidas Jabulani: small ridges or protrusions with 8 panels. (d) Adidas Tango 12: small grip texture with 32 modified panels. 


\section{Ball trajectory simulation}

We conducted a simple 2D flight simulation to compare the effects of the drag coefficients of the Jabulani and the Tango 12 on their flight distance and flight trajectory (Goff and Carré 2009). The occurrence of irregular and unsteady Asai and Kamemoto 2011 flying with no spin or a low-speed spin (Asai and Kamemoto 2011). However, because this study focused on the relationship between the constant resistance of the ball and its flight trajectory, knuckle effects were ignored in the trajectory simulation. In the trajectory simulation we estimated the drag coefficient with respect to the Reynolds number using a cubic curve to calculate the two-dimensional coordinates of the ball. Therefore, we omitted the lift and side forces acting on the ball. We considered the effect of buoyancy on the flight trajectory to be negligible compared to the effect of drag; therefore, we omitted the buoyancy from our calculations. By using the relationship between the Reynolds number and the drag coefficient, which were measured in the wind tunnel experiment, we calculated the initial ball velocities of the two-dimensional flight trajectory to be 17 and $28 \mathrm{~m} / \mathrm{s}$, respectively; the ball was launched at an angle of $25^{\circ}$ in both cases. We also computed the two-dimensional flight trajectories of the Jabulani (initial speed: 17 and $28 \mathrm{~m} / \mathrm{s}$ ) and the Tango 12 (initial speed: 17.4 and $28.7 \mathrm{~m} / \mathrm{s}$ ) under the condition of ball impacts having the same impulse $(17 \mathrm{~m} / \mathrm{s}: 7.45 \mathrm{~kg} / \mathrm{s}$; $28 \mathrm{~m} / \mathrm{s}: 12.26 \mathrm{~kg} / \mathrm{s})$; in doing so, we considered the difference in mass of the Jabulani $(0.438 \mathrm{~kg})$ and the Tango 12 $(0.428 \mathrm{~kg})$.

\section{Extended total distances of panel bonds}

As an index of the surface roughness of the ball, we measured the extended total distances of the panel bonds using a curvimeter (Concurve 10; KOIZUMI Sokki Mfg. Co., Ltd.) (Figure 3).

\section{Results}

Drag force in wind tunnel test

The critical Reynolds number of the Roteiro, Teamgeist II, Jabulani, and Tango was $\sim 2.2 \times 10^{5}\left(C_{d} \approx 0.12\right), \sim 2.8 \times 10^{5}$ $\left(C_{d} \approx 0.13\right), \sim 3.3 \times 10^{5}\left(C_{d} \approx 0.13\right)$, and $\sim 2.4 \times 10^{5}\left(C_{d} \approx\right.$ 0.15 ) (Figure 4 ). The critical Reynolds number obtained

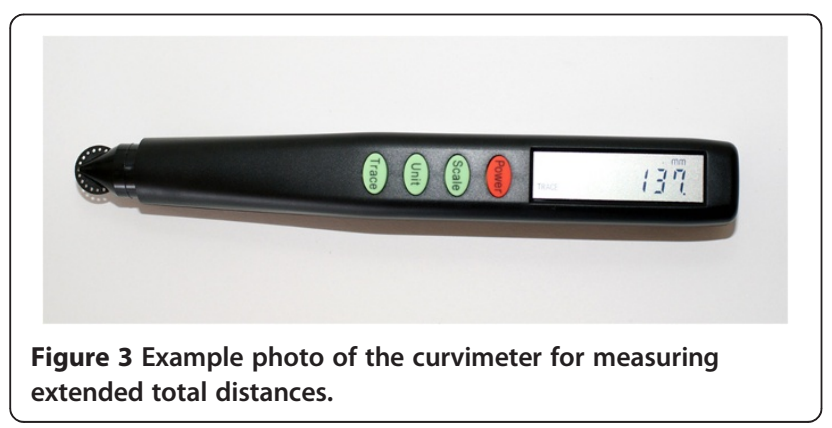

for the Roteiro was the same as that reported by Asai et al. (2007). The standard $C_{d}$ values for the Tango 12 and the Jabulani in the supercritical regime were $\sim 0.18$ and $\sim 0.15$, respectively. The average $C_{d}$ in the subcritical regime was $\sim 0.47$, which was slightly larger than that of the Jabulani ( 0.44). The newer balls showed an increased critical Reynolds number, and the $C_{d}$ curve shifted to the right; however, the $C_{d}$ curve of the Tango 12 was more similar to that of the Roteiro than to that of the Jabulani.

\section{Ball trajectory simulation}

In the ball flight simulation, the flying distances of the Jabulani and the Tango 12 were respectively found to be 17.5 and $19.5 \mathrm{~m}$ for an initial velocity of $17 \mathrm{~m} / \mathrm{s}$ and 47.1 and $44.1 \mathrm{~m}$ for an initial velocity of $28 \mathrm{~m} / \mathrm{s}$, with the ball launching angle being $25^{\circ}$ in both cases (Figure 5). In simulations of ball impacts having the same impulse while considering the mass difference, the flying distances of the Jabulani $(17 \mathrm{~m} / \mathrm{s})$ and the Tango $12(17.4 \mathrm{~m} / \mathrm{s})$ for $7.45 \mathrm{~kg} / \mathrm{s}$ were 17.5 and $20.4 \mathrm{~m}$, respectively. Those of the Jabulani $(28 \mathrm{~m} / \mathrm{s})$ and the Tango $12(28.7 \mathrm{~m} / \mathrm{s})$ for $12.26 \mathrm{~kg} / \mathrm{s}$ were 47.1 and $45.7 \mathrm{~m}$, respectively.

\section{Extended total distances of panel bonds}

The extended total distances of the panel bonds and the number of ball panels were as follows: Adidas Roteiro: $3840 \mathrm{~mm}$, smooth surface with 32 pentagonal and hexagonal panels; Adidas Teamgeist II: 3470 mm, small protuberance with 14 panels; Adidas Jabulani: $1980 \mathrm{~mm}$, small ridges or protrusions with 8 panels; and Adidas Tango 12: $4470 \mathrm{~mm}$, small grip texture with 32 panels. High correlation was observed between the extended total

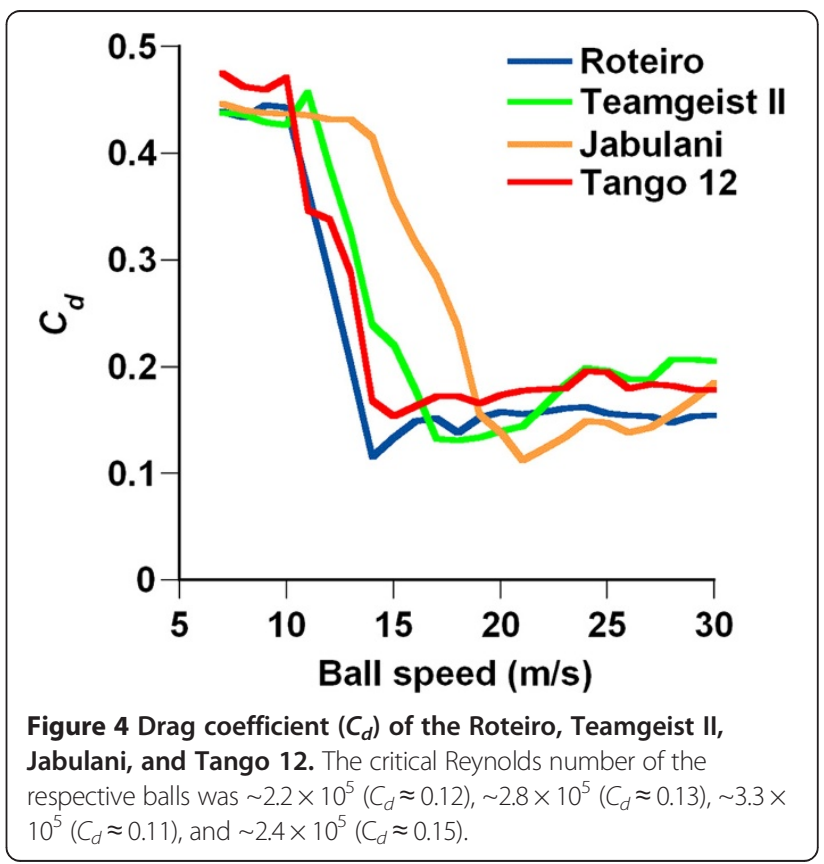


distances of the panel bonds and the critical Reynolds number $(\mathrm{r}=0.9)$ (Figure 6).

\section{Discussion}

Achenbach (1972) claimed that the critical Reynolds number for a smooth sphere is $\sim 3.5 \times 10^{5}$, whereas Bearman and Harvey (1976) reported that the critical Reynolds number of a golf ball is $\sim 6.0 \times 10^{4}$. Therefore, it can be inferred that the critical Reynolds number of the soccer ball used in this study is lesser than that of a smooth sphere and greater than that of a golf ball. Because the Tango 12 has a smaller critical Reynolds number than the Jabulani, it is inferred that the former has lesser aerodynamic resistance than the latter in the medium-speed region $(11<U<$ $19 \mathrm{~m} / \mathrm{s}$ ), the near-critical region for the former. The former has greater aerodynamic resistance than the latter in the high-speed supercritical region $(20<U<29 \mathrm{~m} / \mathrm{s})$.

In the flight trajectory simulation, the Tango 12 flew $2.0 \mathrm{~m}$ farther than the Jabulani when the initial velocity was $17 \mathrm{~m} / \mathrm{s}$, but it flew $3.0 \mathrm{~m}$ lesser when the initial velocity was $28 \mathrm{~m} / \mathrm{s}$. Similarly, in simulations with ball impacts having the same impulse (7.45 and $12.26 \mathrm{~kg} / \mathrm{s})$, the lighter Tango 12 flew farther. In the medium-speed region, where the coefficient of resistance of the Tango 12 was small, the difference in flying distances was as large as $2.9 \mathrm{~m}$. However, in the high-speed region, where the coefficient of resistance of the Tango 12 was large, the difference in flying distances was reduced to $1.4 \mathrm{~m}$.

These results suggest that the Tango 12, one of the newest soccer balls, has less air resistance in the mediumspeed region than the Jabulani and can easily acquire large initial velocity in this region. In other words, this ball can easily gather speed in the frequently used medium-speed range, and therefore, it should be relatively suitable for a passing-based game of soccer.

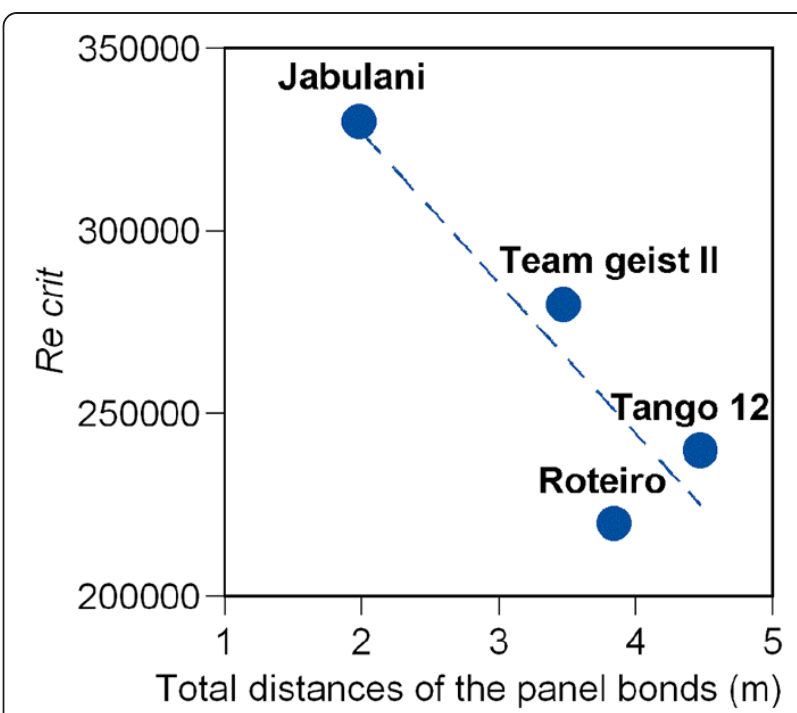

Figure 6 Correlation between the extended total distances of the panel bonds and the critical Reynolds number $(r=0.9)$.

The critical Reynolds number of each ball decreases with the number of panels, i.e. it decreases from the Roteiro to the Teamgeist II to the Jabulani. Furthermore, the extended total distance of the panel bonds decreases with the number of panels. The new Tango 12 ball has 32 panels; therefore, its extended total distance of panel bonds will increase, and its critical Reynolds number will be similar to that of the 32-panel Roteiro ball $(r=0.9)$. Achenbach (1974) reported that an increase in the roughness of the spherical surface decreases the critical Reynolds number. From these points, it can be concluded that the roughness of the ball surface increases with the extended total distance of panel bonds, causing the critical Reynolds number to decrease. In terms of roughness, the panel surface of the Roteiro is relatively smooth; the Teamgeist II has small
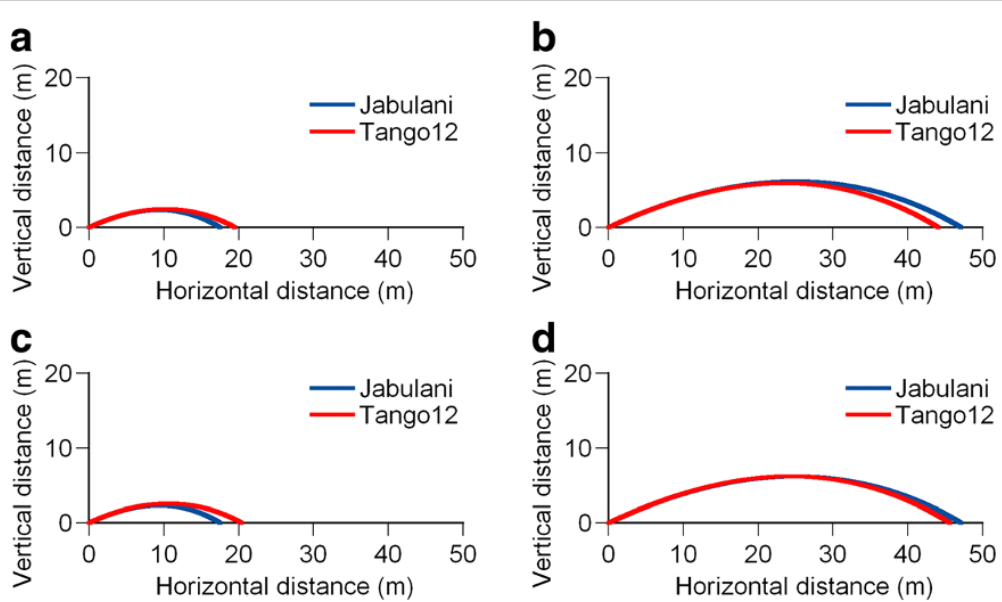

Figure 5 Flight trajectory of the Jabulani and the Tango 12 in a simple 2D flight simulation. (a) Initial ball velocity: $17 \mathrm{~m} / \mathrm{s}$. (b) Initial ball velocity: $28 \mathrm{~m} / \mathrm{s}$. (c) Impulse of ball impact: $7.45 \mathrm{~kg} / \mathrm{s}$. (d) Impulse of ball impact: $12.26 \mathrm{~kg} / \mathrm{s}$. Ball launching angle in all cases: $25^{\circ}$. 
protuberances; the Jabulani has small ridges; and the Tango 12 has small grip textures. Generally, the critical Reynolds number of a sphere decreases as the surface roughness increases. The critical Reynolds number of the Roteiro was lower than that of the Jabulani despite the panel surface of the Roteiro being relatively smoother than that of the Jabulani. The 'small designs' on the soccer ball panels appeared to play a small role in this experiment (see Additional file 1). Therefore, the critical Reynolds number of a soccer ball, as considered within the scope of this experiment, may depend on the extended total distance of the panel bonds rather than the small designs on the panel surfaces.

\section{Conclusion}

This study aims to clarify the drag coefficient and critical Reynolds number of the newest soccer ball-the Adidas Tango 12 (32 panels) - and conventional soccer balls-the Adidas Roteiro (32 panels), Adidas Teamgeist II (14 panels), and Adidas Jabulani (8 panels) - through a wind tunnel experiment. Furthermore, a simple 2D flight trajectory simulation was conducted based on the drag coefficient, and the effect of the drag characteristics on the flight distance and flight trajectory of these balls was examined. The critical Reynolds number of the Roteiro, Teamgeist II, Jabulani, and Tango 12 was $\sim 2.2 \times 10^{5}\left(C_{d} \approx 0.12\right), \sim 2.8 \times 10^{5}\left(C_{d} \approx\right.$ $0.13), \sim 3.3 \times 10^{5}\left(C_{d} \approx 0.13\right)$, and $\sim 2.4 \times 10^{5}\left(C_{d} \approx 0.15\right)$, respectively. The flight trajectory simulation suggests that the Tango 12, one of the newest soccer balls, has less air resistance in the medium-speed region than the Jabulani and can thus easily acquire large initial velocity in this region. The critical Reynolds number of a soccer ball, as considered within the scope of this experiment, may depend on the extended total distance of the panel bonds rather than the small designs on the panel surfaces.

\section{Additional file}

Additional file 1: Drag coefficient (c) of Teamgeist (a) and Teamgeist II (b).

\section{Competing interests}

The authors declare that they have no competing interests.

\section{Authors' contribution}

TA designed the research. TA and KS carried out the experiments. TA and KS analysed data. TA wrote the manuscript. Both authors read and approved the final manuscript.

\section{Author details}

${ }^{1}$ Institute of Health and Sport Sciences, B207, University of Tsukuba, Tsukuba 305-8574, Japan. ${ }^{2}$ Yamagata University, Koshirakawa 1-4-12, Yamagata 990-8560, Japan.

Received: 10 January 2013 Accepted: 16 April 2013

Published: 19 April 2013

\section{References}

Achenbach E (1972) Experiments on the flow past spheres at very high Reynolds numbers. J Fluid Mech 54:565-575

Achenbach E (1974) Vortex shedding from sphere. J Fluid Mech 62:209-221

Asai T, Kamemoto K (2011) Flow structure of knuckling effect in footballs. J Fluid Struct 27:727-733

Asai T, Seo K, Kobayashi O, Sakashita R (2007) Fundamental aerodynamics of the soccer ball. Sports Eng 10:101-109

Bearman PW, Harvey JK (1976) Golf ball aerodynamics. Aeronaut Q 27:112-122

Bray K, Kerwin DG (2003) Modelling the flight of a soccer ball in a direct free kick. J Sports Sci 21:75-85

Davies JM (1949) The aerodynamics of golf balls. J Appl Phys 20:821-828

Goff JE, Carré MJ (2009) Trajectory analysis of a soccer ball. Am J Phys 77:1020-1027

LeRoy W, Alaways LW, Hubbard M (2001) Experimental determination of baseball spin and lift. J Sports Sci 19:349-358

Mehta RD (1985) Aerodynamics of sports balls. Annu Rev Fluid Mech 17:151-189

Mehta RD, Bentley K, Proudlove M, Varty P (1983) Factors affecting cricket ball swing. Nature 303:787-788

Nathan AM, Hopkins J, Lance C, Hank K (2008) The effect of spin on the flight of a baseball. Am J Phys 76:119-124

Smits AJ, Ogg S (2004) Aerodynamics of the Golf Ball. In: Hung GK, Pallis JM (ed) Biomedical Engineering Principles in Sports. Kluwer Academic, New York, pp 3-27

Štěpánek A (1988) The aerodynamics of tennis balls: the topspin lob. Am J Phys 56:138-142

Thompson JJ (1910) The dynamics of a golf ball. Nature 85:2151-2157

Watts RG, Ferrer R (1987) The lateral force on a spinning sphere: aerodynamics of a curveball. Am J Phys 55:40-44

Watts RG, Sawyer E (1975) Aerodynamics of a knuckleball. Am J Phys 43:960-963

Wei Q, Lin R, Liu Z (1988) Vortex-induced dynamics loads on a non-spinning volleyball. Fluid Dyn Res 3:231-237

Zayas JM (1986) Experimental determination of the coefficient of drag of a tennis ball. Am J Phys 54:622-625

doi:10.1186/2193-1801-2-171

Cite this article as: Asai and Seo: Aerodynamic drag of modern soccer balls. SpringerPlus 2013 2:171.

\section{Submit your manuscript to a SpringerOpen ${ }^{\odot}$ journal and benefit from: \\ - Convenient online submission \\ Rigorous peer review \\ - Immediate publication on acceptance \\ - Open access: articles freely available online \\ - High visibility within the field \\ - Retaining the copyright to your article}

Submit your next manuscript at $>$ springeropen.com 